\title{
"Simple" AIEgens for Non-doped Solution-Processed OLEDs with Emission Close to Pure Red in sRGB Gamut
}

Tianfu Zhang, ${ }^{\dagger,}$ Zhicong Zhou, ${ }^{\dagger}+{ }^{\dagger}$ Zheng Zheng,,${ }^{\|} *$ Jianyu Zhang, ${ }^{\dagger}$ Ying Yu, ${ }^{\dagger}$ Jacky W. Y. Lam, $^{\dagger}$ Jonathan E. Halpert ${ }^{\dagger} *$ and Ben Zhong Tang ${ }^{\dagger, \S, \delta, *}$

Mr. T. Zhang, Mr. Z. Zhou, Mr. J. Zhang, Ms. Y. Yu, Dr. J. W. Y. Lam, Prof. J. E. Halpert, Prof. B. Z. Tang

${ }^{\dagger}$ Department of Chemistry, the Hong Kong Branch of Chinese National Engineering Research Center for Tissue Restoration and Reconstruction, Institute for Advanced Study, and Guangdong-Hong Kong-Macro Joint Laboratory of Optoelectronic and Magnetic Functional Materials, The Hong Kong University of Science and Technology, Clear Water Bay, Kowloon, Hong Kong, China

E-mail: jhalpert@ust.hk, tangbenz@ust.hk

These authors contributed equally to this work.

"Dr. Z. Zheng

School of Chemistry and Chemical Engineering, Hefei University of Technology, Hefei 230009, China

E-mail: zzheng@hfut.edu.cn

Prof. B. Z. Tang

${ }^{\S}$ Center for Aggregation-Induced Emission, from Molecular Aggregates, SCUT-HKUST Joint Research Institute, State Key Laboratory of Luminescent Materials and Devices, South China University of Technology, Guangzhou 510640, China

Prof. B. Z. Tang

${ }^{\delta}$ AIE institute, Guangzhou Development Distinct, Huangpu, Guangzhou 510530, China

Keywords: aggregation-induced emission, non-doped OLEDs, red and near-Infrared

Development of simple and efficient red emissive luminogens is desirable for optoelectronic devices. However, this task is challenging due to the limited options for molecular design and the difficulties of synthesis. Red emitting molecules possess large $\pi$-conjugated systems, which permit quenching to occur in the solid state due to the $\pi$ - $\pi$ stacking between molecules and are detrimental to the performance of devices. Furthermore, traditional red emitters usually exhibit emission far from pure red in sRGB gamut. Herein, two red luminogens with aggregation-induced emission (AIE) characteristics based on simple D-A structures are explored. The compounds, abbreviated as DCMa and DCIs, show red emission with high fluorescence quantum yields (QY) of 13.2 and $7.8 \%$ in film state. Efficient non-doped 
solution-processed organic light emitting diodes (OLEDs) with a configuration of ITO/PEDOT:PSS/TFB/DCMA or DCIs/TPBi/LiF/Al are fabricated, which emit red electroluminescence at $652 \mathrm{~nm}$ and $711 \mathrm{~nm}$, respectively. In addition, they exhibit CIE coordinates of $(0.63,0.36)$ and $(0.64,0.35)$, respectively, which are close to the value of the primary red color $(0.63,0.34)$ according to the digital television standard. These results of small molecules DCMa and DCIs suggest future methods for designing a new generation of long wavelength emitters for non-doped, solution-processed OLEDs.

\section{Introduction}

Organic light-emitting diodes (OLEDs) have emerged as one of the most competitive types of optoelectronic devices for next-generation flexible displays and solid-state lighting. ${ }^{[1,2]}$ To achieve full-color devices, light-emitting materials with the three primary colors of blue, green and red, are needed. Compared with the blue and green emitters, development of efficient red emitters still lags behind. The design of red emitters has challenges on various fronts, such as the limited options of emissive materials, complicated processing technologies, stagnant device performance and reduced color fidelity.

To date, most of the reported red emitters are based on lanthanide or transition-metal complexes. ${ }^{[3-6]}$ Although these molecules have been at the forefront of many achievements in OLEDs, with their long wavelengths and even near-infrared emission, the application of transition metal-ligand complexes suffers from several problems such as high cost, and limited options for transition-metal cores and thus a dearth of candidates for improvement. Furthermore, the electroluminescence (EL) of transition-metal complexes based OLEDs is phosphorescent in nature, where the long-lived triplet-state excitons are unstable and are easily quenched by different external factors, such as air, moisture and impurities. These sensitivities also restrict their mass production and inhibit further commercialization. Metalfree organic fluorescent materials, on the other hand, possess relatively higher stability and 
structural variation, both of which could theoretically provide more possibilities for materials development and performance improvement. ${ }^{[7,8]}$ Most of the organic emitters prepared for OLEDs devices so far exhibit emission at shorter wavelengths $(630 \mathrm{~nm})$ and are thus not ready for use in commercial applications. Conventional red luminogens can be constructed by extending the $\pi$-conjugation with planar macrocyclic units. However, such an approach involves some difficult, and long, synthesis steps and demands device fabrication technology due to the poor processibility of these molecules. Introduction of strong intramolecular donoracceptor (D-A) interaction is another commonly used strategy for narrowing the energy gap to impart red emission without the need of synthesizing complex molecular structures by harsh organic reactions. ${ }^{[9]}$ Similar to transition-metal complexes, organic D-A molecules cannot be easily altered due to a restricted choice of electron acceptors. Therefore, it is highly desirable to explore a simple strategy for easy synthesis of efficient D-A emitters for OLED applications.

Another problem associated with conventional organic red emitters is that their strong emission in solution dramatically decreases and can be quenched in concentrated solution or in the solid state. That is due to strong intramolecular charge transfer or serious intermolecular $\pi-\pi$ stacking which causes excitons to decay through the nonradiative relaxation channel. Such an aggregation-caused quenching (ACQ) effect substantially limits their roles and the practical applications as solid-state emitters. To solve this intractable problem in EL devices, several strategies are proposed such as the incorporation of steric hindered moieties or doping with host matrix to hamper the intermolecular interaction, however, new troubles emerge such as inefficient charge transportation, unavoidable performance degradation due to phase separation upon thermal process, complicated device fabrication technology due to the uncertain ratio control of dopant addition and high cost of mass production. ${ }^{[10-12]}$ In terms of device fabrication, it is well-established that the solution processing is the most advantageous fabrication method for OLEDs compared to the evaporative deposition. It shows the merits of 
low cost, dosage saving, simply device fabrication and large-area applications. Organic conjugated polymers are once reported to be overwhelming candidates to possess good solution processability and are demonstrated as a cost-effective approach for mass production. ${ }^{[2,12]}$ However, their relatively low quantum yield and exciton annihilation resulting from kinks in the molecular backbone and strong intermolecular interaction are both problems that have yet to be solved. Hence, how to overcome the dilemma between the fabrication of simple non-doped OLEDs and the prevention of ACQ effect of traditional redemissive materials during solution processing has become a tough question to be solved.

In 2001, Tang and coworkers discovered a group of propeller-like molecules containing rotor structures such as silole and tetraphenylethene, which are non-emissive in dilute solution state but show intense emission upon aggregates formation. ${ }^{[13,14]}$ This interesting phenomenon was coined as aggregation-induced emission (AIE). After many studies, the restriction of intermolecular motion has been proposed as the most commonly accepted interpretation for the AIE effect. Since then, many research groups have been devoted to developing the AIE materials and proposing various mechanisms. ${ }^{[15-17]}$ Because AIE luminogens (AIEgens) exhibit enhanced emission in the solid state, they are regarded as promising and widespread materials for fabricating simple non-doped OLEDs. Recently, more and more red AIEgens have been reported through unremitting efforts and some of them have been applied in OLEDs and other display systems (Scheme 1). ${ }^{[18-25]}$ Unfortunately, although these molecules show long-wavelength EL and high device performance, they need to be prepared via multistep reaction routes. Furthermore, the design strategies of red-emissive AIEgens are mostly based on the large $\mathrm{D}-\pi-\mathrm{A}-\pi-\mathrm{D}$ molecular structures, in which different kinds of electron donors with typical AIE units (e.g. tetraphenylethene) are incorporated to guarantee the performance in solid phase. However, a restrict choice of electron acceptor cores (e.g. benzothiadiazole, BTD) for long emission wavelength limits the structural variation and further development of new emitting materials for OLEDs. Therefore, it is highly desirable to 
explore a new and more efficient molecular system for producing small and "simple" molecules with red emission and AIE characteristics for non-doped, solution-processed OLEDs.

In this work, the photophysical properties of two facilely synthesized red emissive AIEgens (DCMa and DCIs) with simple structures and their promising application in non-doped solution processed OLEDs were explored. DCMa and DCIs are AIE-active and emit bright red emission at 644 and $700 \mathrm{~nm}$ in the film state with high fluorescence quantum yields (QY) of 13.2 and $7.8 \%$, respectively. Non-doped OLEDs using these luminogen as emitters show the EL maximum at 652 and $711 \mathrm{~nm}$ with CIE coordinates of $(0.63,0.36)$ and $(0.64,0.35)$, respectively, both of which are close to the value of primary red color $(0.63,0.34)$ according to the digital television standard (sRGB) that is the most widely used color gamut standards for television monitors, the internet, digital photography and so on. ${ }^{[26]}$ Compared with most of the previously reported red AIE emitters, DCMa and DCIs are constructed with a simple D$\pi-\mathrm{A}$ structure using a diphenylamine groups and electron-rich carbazolyl rings as electron donors and malononitrile or 2-(3,5,5-trimethylcyclohex-2-enylidene)-malononitrile as an electron acceptors, respectively. The associated synthetic routes are much easier and show great potential for mass production. The successful application of small molecule DCMa and DCIs for non-doped solution-processed OLEDs will point to a method for the development of long-wavelength emitters for OLEDs.

\section{Result and Discussion}

\subsection{Photophysical Property}

DCMa and DCIs were prepared according to previously published procedures (Figure 1). ${ }^{[27]}$ Both compounds enjoy good solubility in common organic solvents such as dichloromethane, tetrahydrofuran (THF), acetone and dimethylformamide (DMF) which makes them suitable candidates for producing solution processed OLEDs. The absorption spectra of DCMa and DCIs in DMF show peaks at 468 and $484 \mathrm{~nm}$ with high molar absorptivities of $3.06 \times 10^{4}$ and 
$3.43 \times 10^{4}$, respectively (Figure S1). The AIE characteristics of the molecules were further confirmed by studying their emission behaviors in $\mathrm{DMF} / \mathrm{H}_{2} \mathrm{O}$ mixtures where water is the poor solvent. Taking DCMa as an example, gradual addition of water $(\leq 40 \%)$ into its dilute solution had weakened its PL intensity as shown in Figure 2b. That is because DCMa possesses an electron donor and acceptor in its structure, the enhancement of the solvent polarity upon water addition may strengthen the twisted intramolecular charge transfer (TICT) effect, resulting in emission annihilation. ${ }^{[9,28]}$ Their PL intensities were gradually enhanced when water fraction increased over $50 \%$. In this case, the aggregates of DCMa were formed due to its poor water solubility. Thanks to the RIM mechanism, intramolecular motions of DCMa are restricted at aggregate states which decrease the loss of energy through the nonradiative relaxation pathway and bring back the strong fluorescence. Similar to DCMa, the DCIs displayed obvious AIE property but with a more obviously increasing $\alpha_{\mathrm{AIE}}\left(\alpha_{\mathrm{AIE}}=I / I_{0}\right.$, $\mathrm{I}=$ emission intensity in a DMF/water mixture, $I_{0}=$ emission intensity in DMF solvent) value of more than 100 times, confirming that they show properties consistent with AIE (Figure 2c,

\section{Figure S2).}

Then the PL spectra of the molecules in the crystalline and film states were recorded as shown in Figure 2c and d. In the crystalline state, red emission at 652 and $660 \mathrm{~nm}$ with high QY $\left(\Phi_{\mathrm{F}}\right)$ of 10.0 and $8.8 \%$ were observed for DCMa and DCIs, respectively. In the film state, DCMa emitted at $644 \mathrm{~nm}$ with a $\Phi_{\mathrm{F}}$ of $13.2 \%$ while DCIs emitted at $700 \mathrm{~nm}$ with a good $\Phi_{\mathrm{F}}$ of $7.8 \%$. Compared with the emission peak of AIEgens in the crystalline state, that of DCMa showed a hypochromatic shift in the film state, while a bathochromic shift was observed for DCIs (Table 1). That could be attributed to their different molecular conformation and packing mode and their effect on the two states. The fluorescence decays of DCMa and DCIs in the crystalline crystals and thin films were also studied, revealing lifetime values of 0.89 4.58 ns for two compounds (Table 1, Figure S3). The good photophysical properties of two AIEgens in crystals and film states suggested that further investigation of their potential in 
OLEDs was warranted. It was hoped here that they may solve the trade-off between high performance solid state devices and simplified (e.g. undoped) thin film design.

\subsection{Theoretical Calculation}

To gain deeper insight into the photophysical properties of the molecules, DFT calculations were carried out at PBE0/6-311G(d) level with the solvent model called the Polarizable Continuum Model (PCM), (solvent=acetonitrile) using a suite of Gaussian 09 programs

(Figure 3). The DFT calculations were performed based on the crystal structures of DCMa and DCIs for obtaining the reliable results accord with the practice. As illustrated, the crystal structures adopt non-planar conformations as they are constructed from the twisted diphenylamino unit. Such molecular structures should effectively avoid compact intermolecular $\pi-\pi$ packing in the packed state, thus endowing DCMa and DCIs with high $\Phi_{\mathrm{F}}$ in the crystalline and film states. ${ }^{[29]}$ The molecular orbital density in the highest occupied molecular orbitals (HOMO) is mainly distributed on the donor fraction diphenylamino group and the central carbazole ring, while it is primarily distributed on the acceptor part for the lowest unoccupied molecular orbitals (LUMO). Such an orbital distribution suggests strong intramolecular charge-transfer characteristics of the luminogens. The calculated HOMO and LUMO energy levels are -5.57 and $-2.81 \mathrm{eV}$ for DCMa and -5.43 and $-2.93 \mathrm{eV}$ for DCIs, respectively. In addition, their electrochemical properties were further investigated by cyclic voltammetry $(\mathrm{CV})$. As shown in Figure $\mathbf{3 b}$ and $\mathbf{c}$, HOMO energy levels obtained from the CV diagrams are -5.31 and $-5.19 \mathrm{eV}$ for DCMa and DCIs, respectively. Such values are close to that of poly[(9,9-dioctylfluorenyl-2,7-diyl)-co-(4,4'-(N-(4-sec-butylphenyl)diphenylamine) $]$ (TFB), which is indicative of their good hole-transporting properties. The energy gaps $\left(\mathrm{E}_{\mathrm{g}}\right)$ of DCMa and DCIs estimated from the onset wavelengths of their UV spectra were obtained to be 2.29 and $2.17 \mathrm{eV}$, respectively. Their LUMO can be obtained using the equation: $\mathrm{E}_{\mathrm{LUMO}}=$ $\mathrm{E}_{\mathrm{HOMO}}+\mathrm{E}_{\mathrm{g}}$ and are equal to -3.017 and $-3.019 \mathrm{eV}$, respectively. The high LUMO energy of 
the molecules suggests that they possess the narrow band gap which is consistent with their red and NIR emission.

\subsection{Device Fabrication}

Prior to devices fabrication, the thermal properties of DCMa and DCIs were studied by thermogravimetric analysis (TGA) under nitrogen at a heating rate of $10{ }^{\circ} \mathrm{C} \cdot \mathrm{min}^{-1}$. As depicted in Figure S4 and Table S1, DCMa and DCIs lose $5 \%$ of their weight at high temperatures of 200 and $298{ }^{\circ} \mathrm{C}$, confirming that their good thermal stability is suitable for various methods of film fabrication, including vapor deposition. The efficient solid-state emission (from film and crystalline states) and high thermal stability of DCMa and DCIs make them promising materials for fabricating non-doped EL devices by solution processed techniques. According to their energy levels, DCMa and DCIs with a configuration of indium tin oxide (ITO)/poly(3,4-ethylenedioxythiophene) polystyrene sulfonate (PEDOT:PSS) (40 nm)/poly[(9,9-dioctylfluorenyl-2,7-diyl)-co-(4,4'-(N-(4-sec-butylphenyl)diphenylamine)]

(TFB) $\quad(20 \quad \mathrm{~nm}) / \mathrm{emitting} \quad$ layer $\quad(\mathrm{EML}) / 2,2^{\prime}, 2^{\prime \prime}-(1,3,5$-benzinetriyl)-tris(1-phenyl-1-Hbenzimidazole) $(\mathrm{TPBi})(40 \mathrm{~nm}) / \mathrm{LiF}(1 \mathrm{~nm}) / \mathrm{Al}(100 \mathrm{~nm})$ were fabricated (Figure 4a, Figure S6). In these devices, the neat films of DCMa or DCIs serve as an emitting layer (EML), while PEDOT:PSS functions as a hole-injection layer, TFB works as a hole-transport layer (HTL) and TPBi works as an electron transport layer (ETL) as well as the hole blocking layer due to their much lower HOMO values (with the large energy barrier exists between the EML and ETL layers) than that of DCMa and DCIs. The performances of the devices are shown in Figure 4b, Figure S7 and 8. Two devices were turned on at a low bias of 5.0 and $6.6 \mathrm{~V}$, emitting deep red and NIR EL at 652 and $711 \mathrm{~nm}$, respectively, with high stabilities under varying voltage as shown in Figure S5. The maximum EQE values were calculated to be $1.95 \%$ and $0.58 \%$ for devices incorporating DCMa and DCIs, respectively (Table S2). The CIE coordinates of the EL emission are $(0.63,0.36)$ and $(0.64,0.35)$, respectively, which are 
very close to the primary red color of $(0.63,0.34)$ (sRGB/Rec. 601/ Rec. 709$)$ for Digital Standard Definition Television. Their performance in expressing the red primary chromaticity makes them the suitable candidates for commercial digital displays and among most of the previously reported molecular red emitters (Figure 4). ${ }^{[19,22,23,25,30-36]}$ The high performance of the devices thanks to not only the excellent photophysical properties of DCMa and DCIs in film states but also their good hole-transporting as well as electron transporting properties.

\section{Conclusion}

In summary, two red and NIR AIEgens DCMa and DCIs with simple D- $\pi-A$ structures were found to be efficient non-doped red emitters for solution processed OLEDs. DCMa and DCIs exhibit high QY of $13.2 \%$ and $7.8 \%$ in the thin film states as well as good thermal stability in the solid crystalline states. Simple and efficient non-doped OLEDs were fabricated and exhibited long EL wavelength at $625 \mathrm{~nm}$ and $711 \mathrm{~nm}$, respectively. They display good device performance with maximum EQE of up to $1.95 \%$. In addition, devices using these materials show red emission with CIE coordinates of $(0.63,0.36)$ and $(0.64,0.35)$, both of which are close to primary red color $(0.63,0.34)$ according to the digital television standard. The successful application of small molecules DCMa and DCIs for non-doped solution-processed OLEDs devices points to a new strategy for the development of long-wavelength OLEDs emitters. Next generation of AIEgens with simple D-A structure and good film-forming properties are thereby expected to be explored for future OLED devices with better performance..

\section{Experimental Section}

Materials and Characterization: Solvents were all purchased from J\&K, Sigma, Meryer, and AIEgen Biotech and used as received without further purification. Toluene was distilled using sodium as a drying agent and benzophenone as an indicator under nitrogen prior to use. PEDOT:PSS (Al 4083) was purchased from Ossila. Poly[(9,9-dioctylfluorenyl-2,7-diyl)-co(4,4'-(N-(4-sec-butylphenyl) diphenylamine)] (TFB) and 1,3,5-tris (2-N- 
phenylbenzimidazolyl) benzene (TPBi) were purchased from Luminescence Technology Corp. Lithium fluoride (LiF, $\geq 99.99 \%$ ) was purchased from Sigma Aldrich.

UV-vis spectra were measured on a Milton Roy Spectronic 3000 Array spectrophotometer. Steady-state photoluminescence (PL) spectra were measured on a PerkinElmer LS 55 spectrofluorometer. QY was determined by a Quanta- $\varphi$ integrating sphere. Fluorescence lifetimes were measured with Hamamatsu Quantaurus-Tau C11367-11 at room temperature. Electrochemical measurements were recorded on a CHI610D electrochemical workstation in a three-electrode cell using a platinum button as the working electrode, a platinum wire as the counter electrode, and a saturated calomel electrode as the reference electrode in acetonitrile $\left(\mathrm{CH}_{3} \mathrm{CN}\right)$ with $0.1 \mathrm{M} \mathrm{Bu} \mathrm{NPF}_{6}$ at a scan rate of $100 \mathrm{mV} \cdot \mathrm{s}^{-1}$ and ferrocene as the internal standard. TGA measurements were carried out on a TA Q5000 instrument under a dry nitrogen flow at a heating rate of $10^{\circ} \mathrm{C} \cdot \mathrm{min}^{-1}$. Optimized geometric structures in the gas phase of DCMa and DCIs were calculated at the PBE0/6-311G(d) level with solvent model of PCM, (solvent = acetonitrile) by using Gaussian 09 program. Scanning electron microscopy (SEM) micrographs were collected on a field emission scanning electron microscope (FESEM, JEOL-7100F).

Device Fabrication: OLEDs were fabricated on glass substrates coated with patterned ITO. The ITO substrates were ultrasonically cleaned with $2 \%$ micro 90, DI water, acetone, and isopropanol for $20 \mathrm{~min}$ each, followed by $\mathrm{O}_{2}$ plasma cleaning for $30 \mathrm{~min}$. PEDOT:PSS (Al 4083) was filtered by a $0.45 \mu \mathrm{m}$ PVDF filter and spin coated at $3500 \mathrm{rpm}$ for $40 \mathrm{~s}$ onto the ITO substrate and then annealed at $150{ }^{\circ} \mathrm{C}$ for $20 \mathrm{~min}$ in ambient conditions to yield a 40-nmthick thin film. After the annealing of PEDOT:PSS, the following processes were all performed under nitrogen atmosphere. A solution of TFB in anhydrous toluene with a concentration of $12 \mathrm{mg} \cdot \mathrm{ml}^{-1}$ was spin coated at $3000 \mathrm{rpm}$ for $40 \mathrm{~s}$ onto PEDOT:PSS layer and then annealed at $200{ }^{\circ} \mathrm{C}$ for 30 min to form a 20 -nm-thick layer. Emissive layer of DCMa and DCIs with a concentration of $10 \mathrm{mmol} \cdot \mathrm{mL}^{-1}$ in anhydrous THF was spin coated at $3000 \mathrm{rpm}$ 
for $40 \mathrm{~s}$ onto TFB layer, followed by annealing at $100{ }^{\circ} \mathrm{C}$ for $20 \mathrm{~min}$ to form an emissive layer of around 80 and $90 \mathrm{~nm}$, respectively. Next, 1,3,5-tris (2-N-phenylbenzimidazolyl) benzene (TPBi) $(40 \mathrm{~nm}), \mathrm{LiF}(1 \mathrm{~nm})$ and $\mathrm{Al}(100 \mathrm{~nm})$ were deposited successively onto the sample through a shadow mask by thermal evaporation under high vacuum of $1.5 \times 10^{-6}$ Torr. After assembly of electrical contacts, OLEDs with device area of $6 \mathrm{~mm}^{2}$ were ready for characterization. All measurements were conducted in ambient air. The active device area of each device was $2.0 \times 3.0 \mathrm{~mm}^{2}$. A Keithley $2401 \mathrm{SMU}$ instrument was used as a power source for the OLEDs. All the OLEDs were characterized using a QE Pro Ocean Optics spectrometer connected with a $400 \mu \mathrm{m}$ optical fiber (QP400-2-SR-BX). The optical fiber was carefully calibrated with an Ocean Optics HL-3 plus VIS-NIR light source.

\section{Supporting Information}

Supporting Information is available.

\section{Acknowledgements}

This work was financially supported by the National Natural Science Foundation of China (21788102, 21490570, and 21490574), the Research Grant Council of Hong Kong (16305518, 16305618, and C6009-17G), the Innovation and Technology Commission (ITCCNERC14SC01), and the Natural Science Foundation of Guangdong Province (2019B121205002 and 2019B030301003).

\section{References}

[1] Tang, C. W.; VanSlyke, S. A. Appl. Phys. Lett. 1987, 51, 913.

[2] Tang, C. W.; VanSlyke, S. A.; Chen, C. H. J. Appl. Phys. 1989, 65, 3610.

[3] Cao, X.; Miao, J.; Zhu, M.; Zhong, C.; Yang, C.; Wu, H.; Qin, J.; Cao, Y. Chem.

Mater. 2015, 27, 96.

[4] Graham, K. R.; Yang, Y.; Sommer, J. R.; Shelton, A. H.; Schanze, K. S.; Xue, J.;

Reynolds, J. R. Chem. Mater. 2011, 23, 5305.

[5] Sakakibara, Y.; Okutsu, S.; Enokida, T.; Tani, T. Appl. Phys. Lett. 1999, 74, 2587. 
[6] Tuong Ly, K.; Chen-Cheng, R.-W.; Lin, H.-W.; Shiau, Y.-J.; Liu, S.-H.; Chou, P.-T.;

Tsao, C.-S.; Huang, Y.-C.; Chi, Y. Nat. Photonics 2017, 11, 63.

[7] Gross, M.; Müller, D. C.; Nothofer, H.-G.; Scherf, U.; Neher, D.; Bräuchle, C.;

Meerholz, K. Nature 2000, 405, 661.

[8] Chen, C.-T. Chem. Mater. 2004, 16, 4389.

[9] Zhao, Z.; Su, H.; Zhang, P.; Cai, Y.; Kwok, R. T. K.; Chen, Y.; He, Z.; Gu, X.; He, X.; Sung, H. H. Y.; Willimas, I. D.; Lam, J. W. Y.; Zhang, Z.; Tang, B. Z. J. Mater. Chem. B 2017, 5,1650 .

[10] Qiu, Y.; Wei, P.; Zhang, D. Q.; Qiao, J.; Duan, L.; Li, Y. K.; Gao, Y. D.; Wang, L. D. Adv. Mater. 2006, 18, 1607.

[11] Guo, J.; Li, X.-L.; Nie, H.; Luo, W.; Gan, S.; Hu, S.; Hu, R.; Qin, A.; Zhao, Z.; Su, S.J.; Tang, B. Z. Adv. Funct. Mater. 2017, 27, 1606458.

[12] Zhao, Y.; Chen, J.; Ma, D. ACS Appl. Mater. Interfaces 2013, 5, 965.

[13] Zhao, Z.; Zhang, H.; Lam, J. W. Y.; Tang, B. Z. Angew. Chemie Int. Ed. 2020, 59, 9888.

[14] Luo, J.; Xie, Z.; Lam, J. W. Y.; Cheng, L.; Chen, H.; Qiu, C.; Kwok, H. S.; Zhan, X.; Liu, Y.; Zhu, D.; Tang, B. Z. Chem. Commun. 2001, 18, 1740.

[15] Zhang, T.; Li, Y.; Zheng, Z.; Ye, R.; Zhang, Y.; Kwok, R. T. K.; Lam, J. W. Y.; Tang, B. Z. J. Am. Chem. Soc. 2019, 141, 5612.

[16] Zhang, H.; Zhao, Z.; Turley, A. T.; Wang, L.; McGonigal, P. R.; Tu, Y.; Li, Y.; Wang, Z.; Kwok, R. T. K.; Lam, J. W. Y.; Tang, B. Z. Adv. Mater. 2020, 32, 2001457.

[17] Nie, H.; Hu, K.; Cai, Y.; Peng, Q.; Zhao, Z.; Hu, R.; Chen, J.; Su, S.-J.; Qin, A.; Tang, B. Z. Mater. Chem. Front. 2017, 1, 1125.

[18] Wan, Q.; Tong, J.; Zhang, B.; Li, Y.; Wang, Z.; Tang, B. Z. Adv. Opt. Mater. 2020, 8, 1901520. 
[19] Yu, Y.; Xu, Z.; Zhao, Z.; Zhang, H.; Ma, D.; Lam, J. W. Y.; Qin, A.; Tang, B. Z. ACS Omega 2018, 3, 16347.

[20] Ravindran, E.; Somanathan, N. J. Mater. Chem. C 2017, 5, 7436.

[21] Huang, J.; Qiao, X.; Xia, Y.; Zhu, X.; Ma, D.; Cao, Y.; Roncali, J. Adv. Mater. 2008, $20,4172$.

[22] Lee, W. W. H.; Zhao, Z.; Cai, Y.; Xu, Z.; Yu, Y.; Xiong, Y.; Kwok, R. T. K.; Chen, Y.; Leung, N. L. C.; Ma, D.; Lam, J. W. Y.; Qin, A.; Tang, B. Z. Chem. Sci. 2018, 9, 6118. [23] Zhao, Z.; Geng, J.; Chang, Z.; Chen, S.; Deng, C.; Jiang, T.; Qin, W.; Lam, J. W. Y.; Kwok, H. S.; Qiu, H.; Liu, B.; Tang, B. Z. J. Mater. Chem. 2012, 22, 11018.

[24] Li, H.; Chi, Z.; Zhang, X.; Xu, B.; Liu, S.; Zhang, Y.; Xu, J. Chem. Commun. 2011, 47, 11273.

[25] Qin, W.; Lam, J. W. Y.; Yang, Z.; Chen, S.; Liang, G.; Zhao, W.; Kwok, H. S.; Tang, B. Z. Chem. Commun. 2015, 51, 7321.

[26] DeMarsh, L. SMPTE J. 1993, 102, 1095.

[27] Zheng, Z.; Zhang, T.; Liu, H.; Chen, Y.; Kwok, R. T. K.; Ma, C.; Zhang, P.; Sung, H. H. Y.; Williams, I. D.; Lam, J. W. Y.; Tang, B. Z. ACS Nano 2018, 12, 8145.

[28] Hu, R.; Lager, E.; Aguilar-Aguilar, A.; Liu, J.; Lam, J. W. Y.; Sung, H. H. Y.; Williams, I. D.; Zhong, Y.; Wong, K. S.; Peña-Cabrera, E.; Tang, B. Z. J. Phys. Chem. C 2009, 113,15845 .

[29] Qiu, Z.; Zhao, W.; Cao, M.; Wang, Y.; Lam, J. W. Y.; Zhang, Z.; Chen, X.; Tang, B. Z. Adv. Mater. 2018, 30, 1803924.

[30] Chen, P.; Wang, L.-P.; Tan, W.-Y.; Peng, Q.-M.; Zhang, S.-T.; Zhu, X.-H.; Li, F. ACS Appl. Mater. Interfaces 2015, 7, 2972.

[31] Sun, K.; Chu, D.; Cui, Y.; Tian, W.; Sun, Y.; Jiang, W. Org. Electron. 2017, 48, 389.

[32] Sun, K.; Sun, Y.; Liu, D.; Feng, Y.; Zhang, X.; Sun, Y.; Jiang, W. Dye. Pigment. 2017, $147,436$. 
[33] Thangthong, A.; Prachumrak, N.; Saengsuwan, S.; Namuangruk, S.; Keawin, T.;

Jungsuttiwong, S.; Sudyoadsuk, T.; Promarak, V. J. Mater. Chem. C 2015, 3, 3081.

[34] Li, Y.; Wang, W.; Zhuang, Z.; Wang, Z.; Lin, G.; Shen, P.; Chen, S.; Zhao, Z.; Tang, B. Z. J. Mater. Chem. C 2018, 6, 5900.

[35] Khanasa, T.; Prachumrak, N.; Rattanawan, R.; Jungsuttiwong, S.; Keawin, T.;

Sudyoadsuk, T.; Tuntulani, T.; Promarak, V. Chem. Commun. 2013, 49, 3401.

[36] Huang, J.; Liu, Q.; Zou, J.-H.; Zhu, X.-H.; Li, A.-Y.; Li, J.-W.; Wu, S.; Peng, J.; Cao, Y.; Xia, R.; Bradley, D. D. C.; Roncali, J. Adv. Funct. Mater. 2009, 19, 2978.
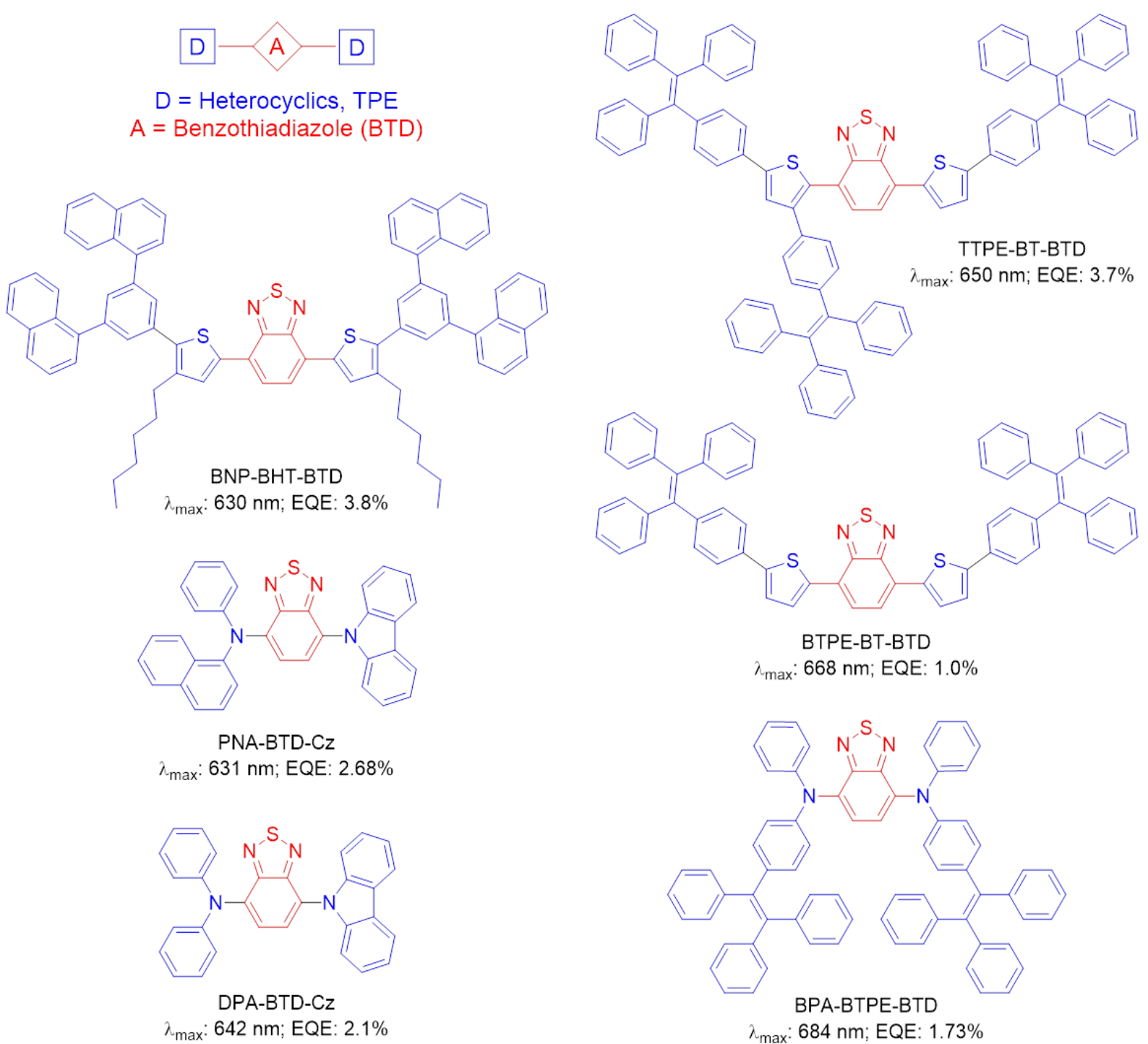

Scheme 1. Chemical structures of previously reported red AIEgens. 
a
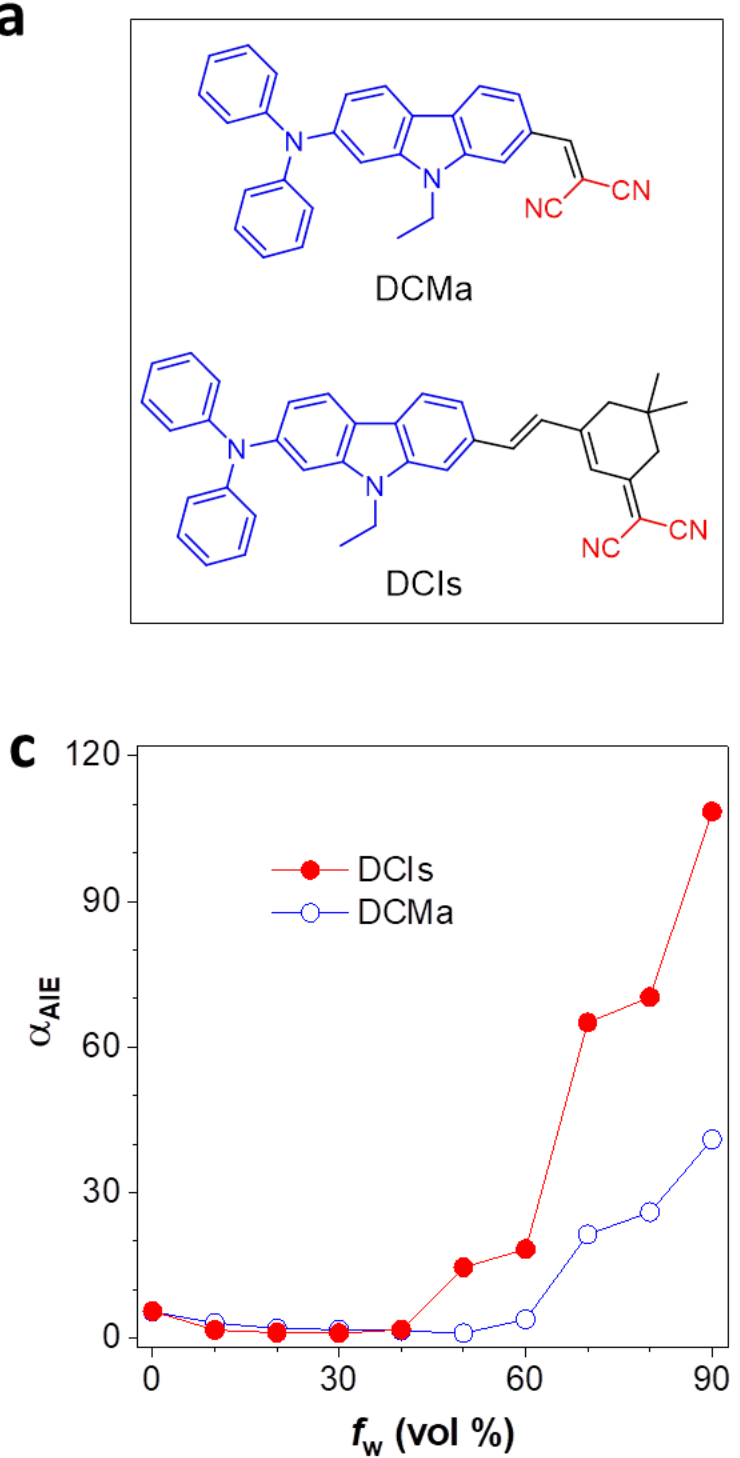
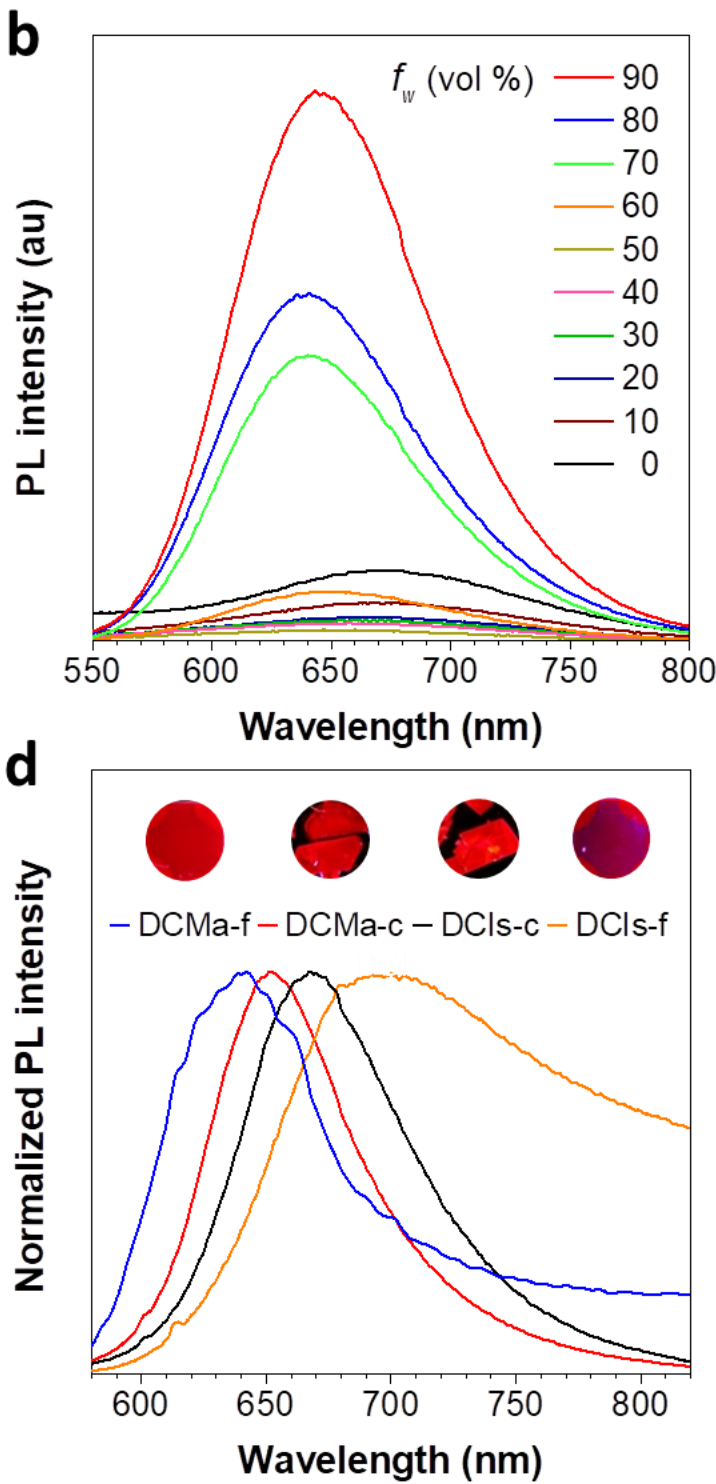

Figure 1. (a) Chemical structures of DCMa and DCIs. (b) PL spectra of DCMa in DMF/water mixtures with different water fractions $\left(f_{\mathrm{w}}\right)$. (c) AIE plots of DCMa and DCIs; $\alpha_{\mathrm{AIE}}=I / I_{0}, I=$ PL intensity in a DMF/water mixture, $I_{0}=\mathrm{PL}$ intensity in DMF solution; concentration: $10^{-5}$ M. (d) Normalized PL spectra of DCMa and DCIs in crystalline (c) and film (f) states. Inset: fluorescent photographs of the AIEgens in crystalline and film states taken under $365 \mathrm{~nm} \mathrm{UV}$ irradiation.

Table 1. The photophysical data of DCMa and DCIs.

\begin{tabular}{cccccccccc}
\hline & & $\varepsilon$ & \multicolumn{2}{c}{$\lambda_{\mathrm{em}}[\mathrm{nm}]^{\mathrm{c})}$} & & $\Phi_{\mathrm{F}}[\%]^{\mathrm{d})}$ & \multicolumn{2}{c}{$\tau[\mathrm{ns}]^{\mathrm{e})}$} \\
\cline { 4 - 10 } AlEgen & $\lambda_{\text {abs }}[\mathrm{nm}]^{\mathrm{a})}$ & {$\left[10^{4} \mathrm{~L} \cdot \mathrm{mol}^{-1} \cdot \mathrm{cm}^{-1}\right]^{\mathrm{b})}$} & Crystal & Film & Crystal & Film & Crystal & Film \\
\hline DCMa & 468 & 3.06 & 652 & 644 & 10.0 & 13.2 & 4.58 & 1.12 \\
DCls & 484 & 3.43 & 660 & 700 & 8.8 & 7.8 & 0.89 & 1.14 \\
\hline
\end{tabular}

a) Maximum absorption peak, concentration: $10^{-5} \mathrm{M}$ in DMF;

b) Molar absorptivity in DMF solution;

c) Maximum emission wavelength.

d) Absolute fluorescence quantum yield measured using an integrating sphere.

e) Fluorescence lifetime. 
a

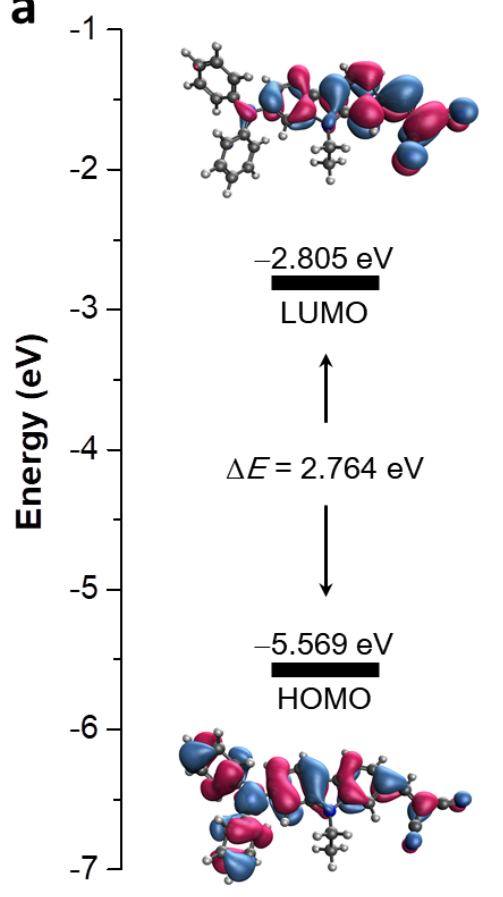

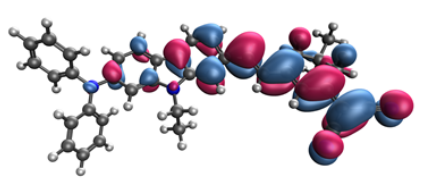

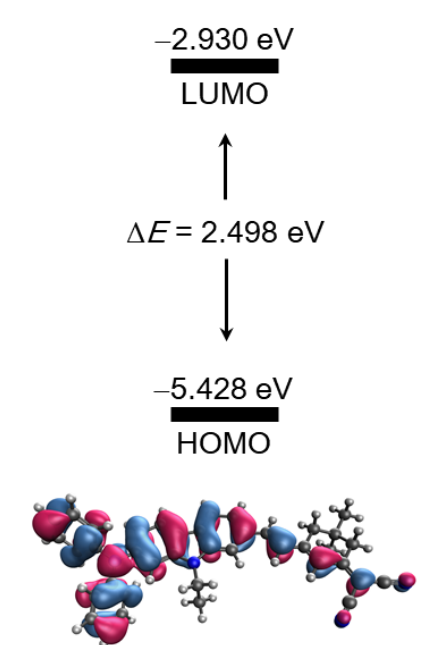

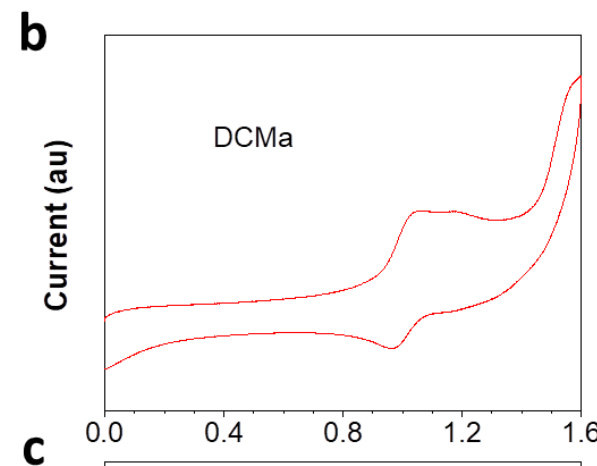

C

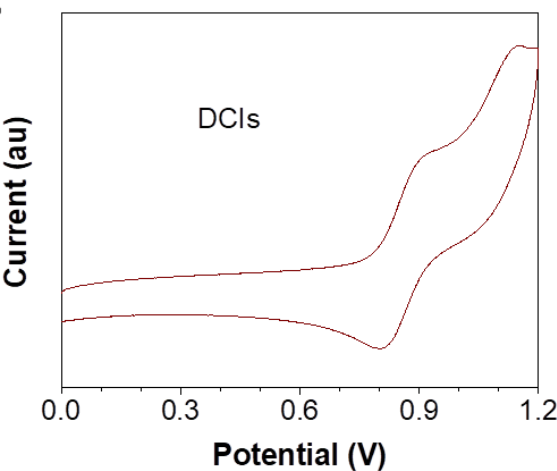

Figure 2. (a) The electron density distribution of frontier orbitals and energy levels of DCMa and DCIs calculated using DFT at PBE0/6-311G(d) level with solvent model of PCM (solvent $=$ acetonitrile), Gaussian 09 program. Cyclic voltammograms of (b) DCMa and (c) DCIs films on a platinum electrode in a $0.1 \mathrm{M}$ solution of $\mathrm{Bu}_{4} \mathrm{NPF}_{6}$ in acetonitrile at a scan rate of 100 $\mathrm{mV} \cdot \mathrm{s}^{-1}$. 

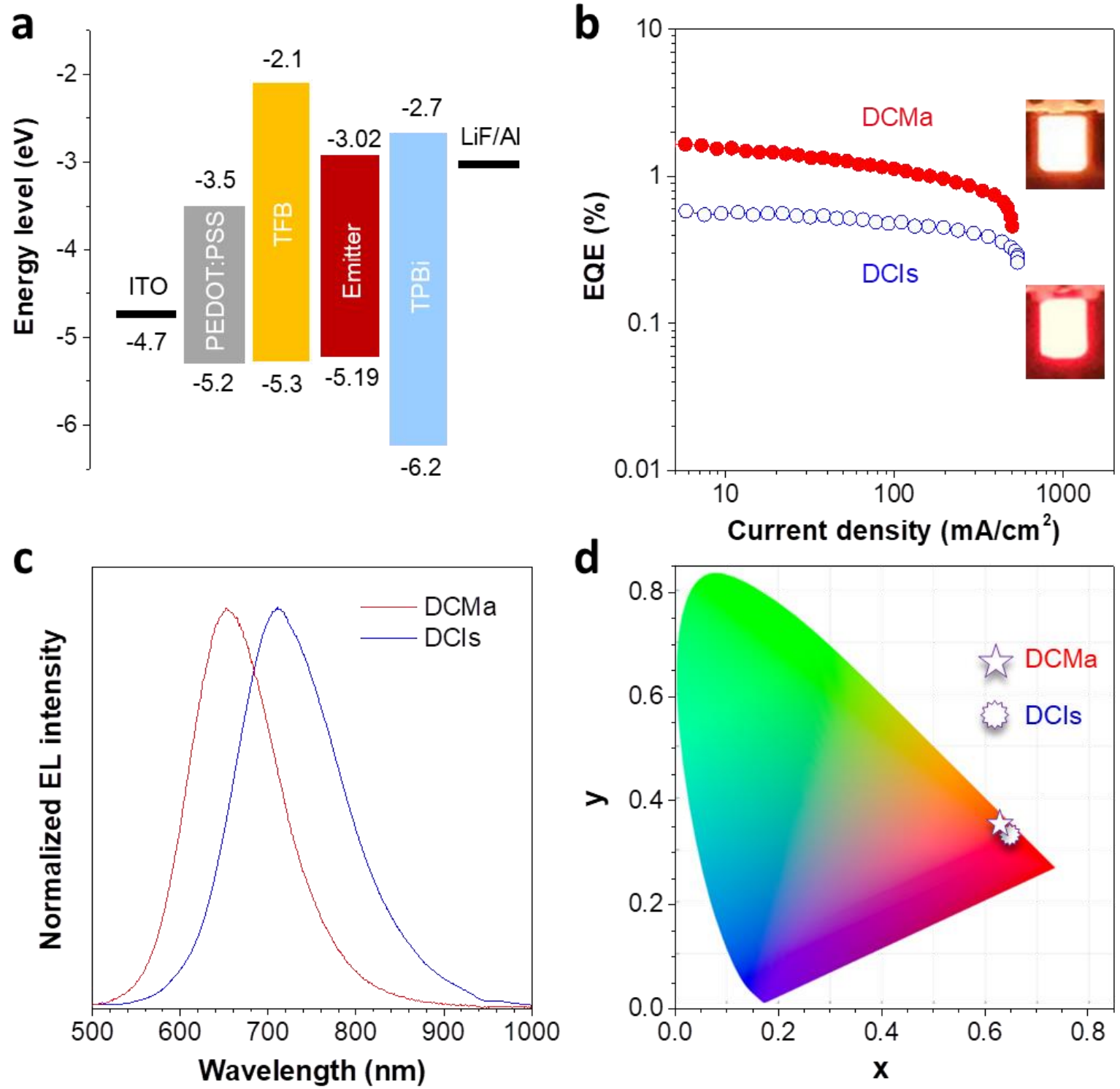

Figure 3. (a) Device configuration for an OLED using DCIs as emitter: ITO/PEDOT:PSS (40 $\mathrm{nm}) / \mathrm{TFB}(20 \mathrm{~nm}) / \mathrm{DCIs}(90 \mathrm{~nm}) / \mathrm{TPBi}(40 \mathrm{~nm}) / \mathrm{LiF}(1 \mathrm{~nm}) / \mathrm{Al}(100 \mathrm{~nm})$. For device based on DCMa, the thickness of its emitting layer is $80 \mathrm{~nm}$. (b) External quantum efficiency (EQE) of OLED using DCMa or DCIs as emitter. Insets: photos of devices based on DCMa (upper) and DCIs (lower). (c) Electroluminescence (EL) spectra of DCMa and DCIs devices. (d) CIE 1931 diagram of DCMa and DCIs. 


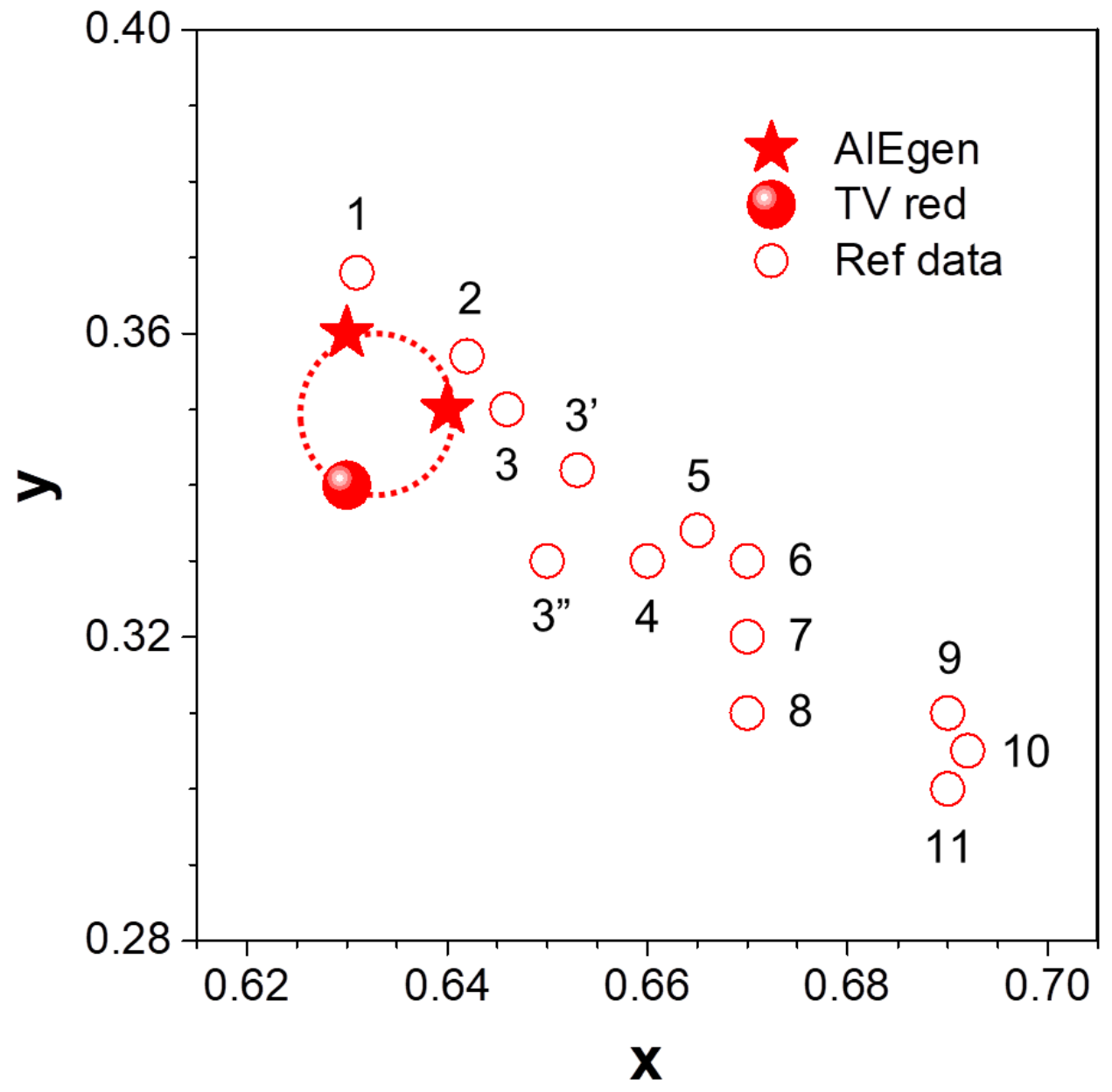

Figure 4. Comparison between the CIE values of these and previously reported red lightemitting electroluminescence devices. Emitters used in the previously reported OLED devices: 1 = PNA-BTD-Cz, $2=$ BNP-BTD-Cz, $3=$ BNP-BBOBT-BTD $(3=$ device I, 3' = device II, $3 "=$ device III), $4=$ CAPTB, $5=$ t-BPiTBT-TPATPE, $6=$ T5B, $7=$ TTPE-BTBTD, 8 = MPPA-MCBP, 9 = MPPA:MCBP, $10=$ BPA-BTPE-BTD, $11=$ MPPA-3Cz (see references for previously reported device data). 
"Simple" AIEgens for Non-doped Solution-Processed OLEDs with Emission Close to Pure Red in sRGB Gamut

Tianfu Zhang, $†$ Zhicong Zhou, $†$ Zheng Zheng, * Jianyu Zhang, Ying Yu, Jacky W. Y. Lam, Jonathan E. Halpert* and Ben Zhong Tang*

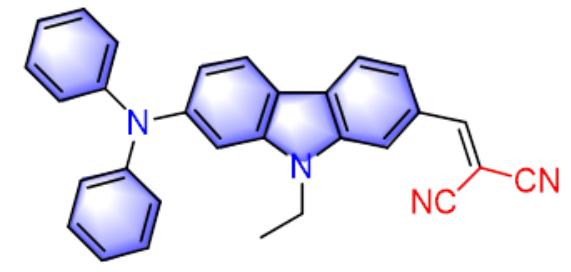

DCMa

$\lambda_{\max }: 652 \mathrm{~nm}, \mathrm{CIE}: 0.63,0.36$

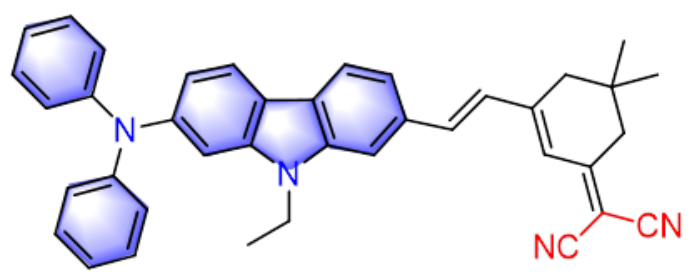

$\mathrm{DCls}$

$\lambda_{\max }: 711 \mathrm{~nm}, \mathrm{CIE}: 0.64,0.35$

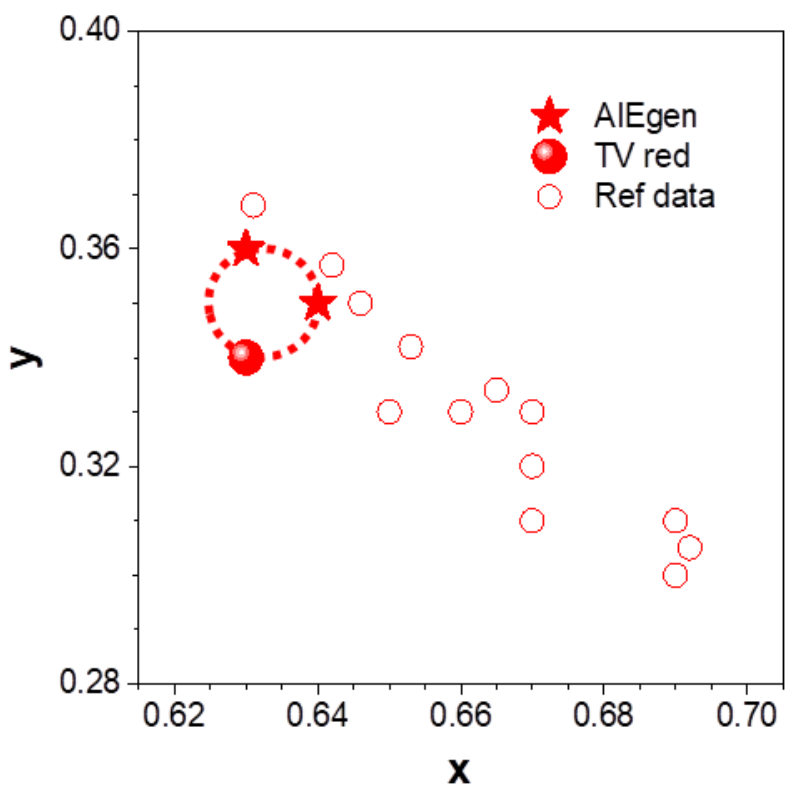

ToC figure 
(C) Copyright 2020. WILEY-VCH GmbH.

Supporting Information

"Simple" AIEgens for Non-doped Solution-Processed OLEDs with Emission Close to Pure Red in sRGB Gamut

Tianfu Zhang, $†$ Zhicong Zhou, $†$ Zheng Zheng, * Jianyu Zhang, Ying Yu, Jacky W. Y. Lam, Jonathan E. Halpert* and Ben Zhong Tang*

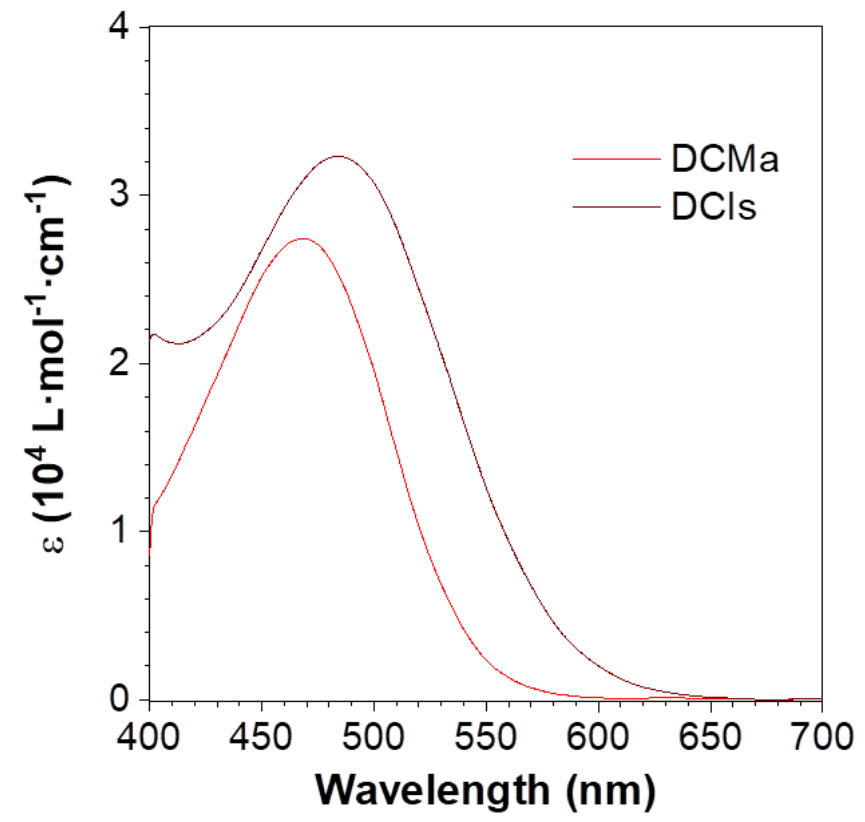

Figure S1. Molar absorptivity spectra of DCMa and DCIs in DMF.
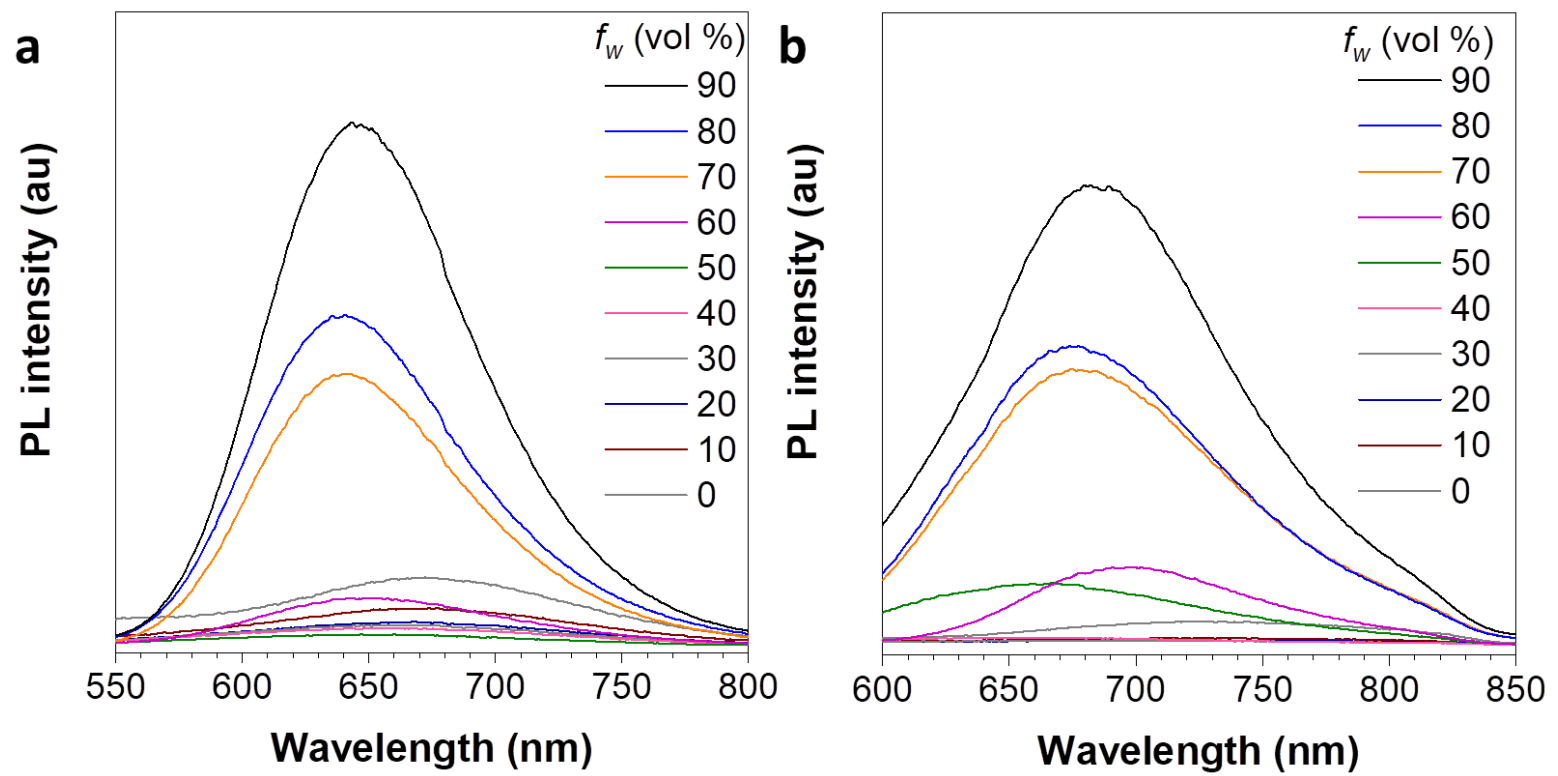

Figure S2. PL spectra of DCMa and DCIs in DMF/water mixtures with different water fractions $\left(f_{\mathrm{w}}\right.$ from 0 to $\left.90 \mathrm{vol} \%\right)$. 


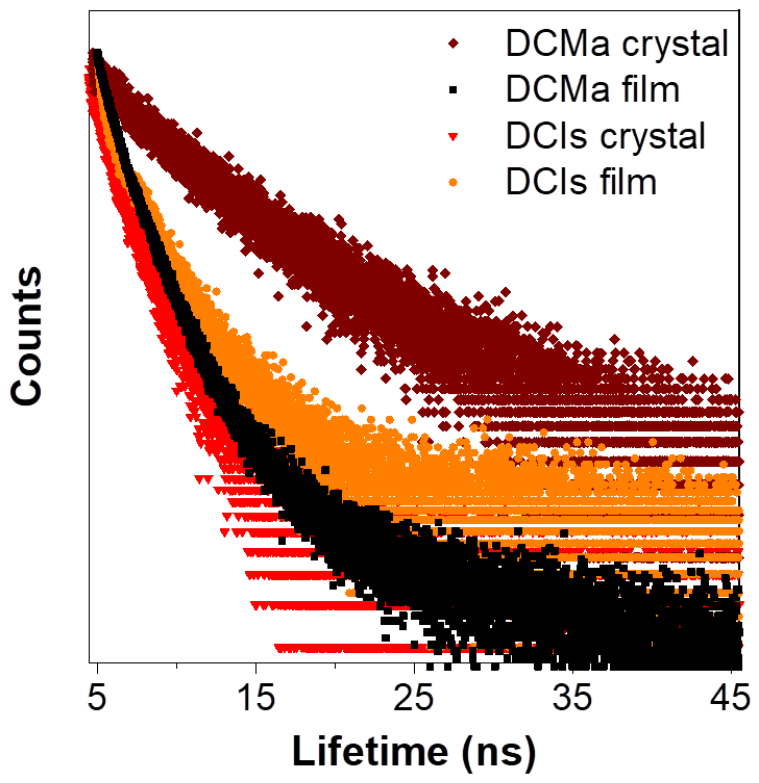

Figure S3. Fluorescence decay curves of DCMa and DCIs in crystalline and film states.

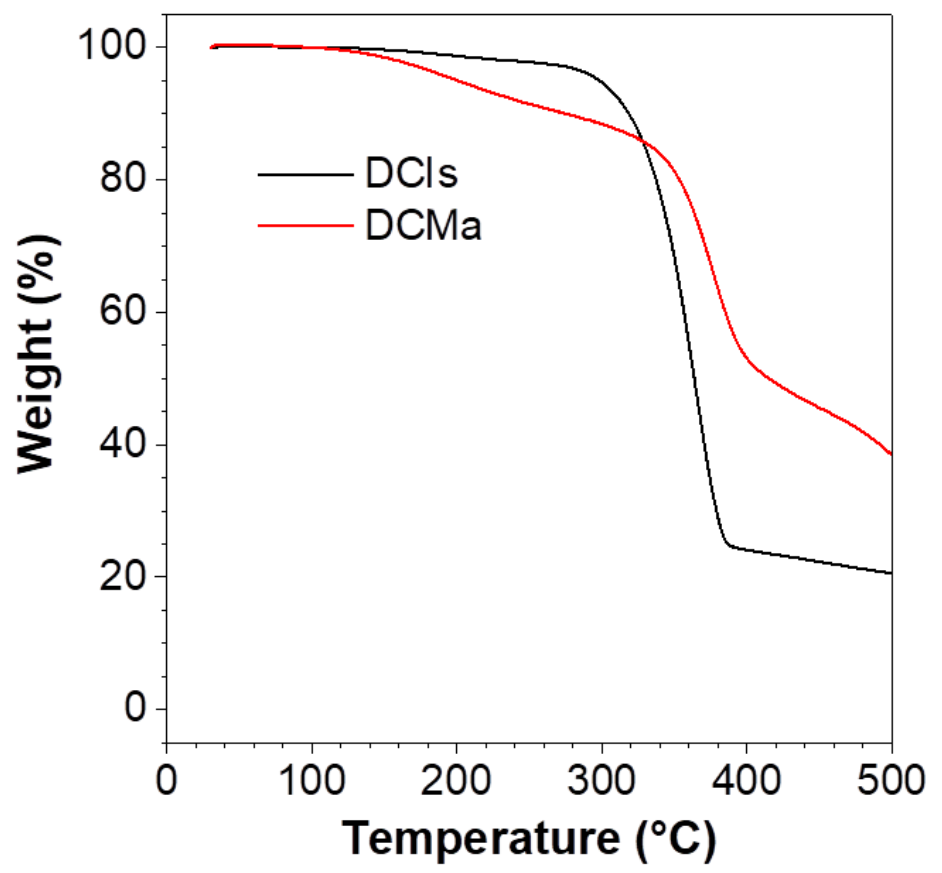

Figure S4. TGA thermograms of DCMa and DCIs. The data were recorded under $\mathrm{N}_{2}$ at a heating rate of $10^{\circ} \mathrm{C} \mathrm{min}^{-1}$.

Table S1. Physical properties of DCMa and DCIs.

\begin{tabular}{ccccc}
\hline Compounds & $\mathrm{Td}_{\mathrm{d}}\left[{ }^{\circ} \mathrm{C}\right]$ & $\mathrm{HOMO}[\mathrm{eV}]$ & LUMO $[\mathrm{eV}]$ & $\mathrm{E}_{\mathrm{g}}[\mathrm{eV}]$ \\
\hline DCMa & 200 & -5.31 & -3.02 & 2.29 \\
DCls & 298 & -5.19 & -3.02 & 2.17 \\
\hline
\end{tabular}



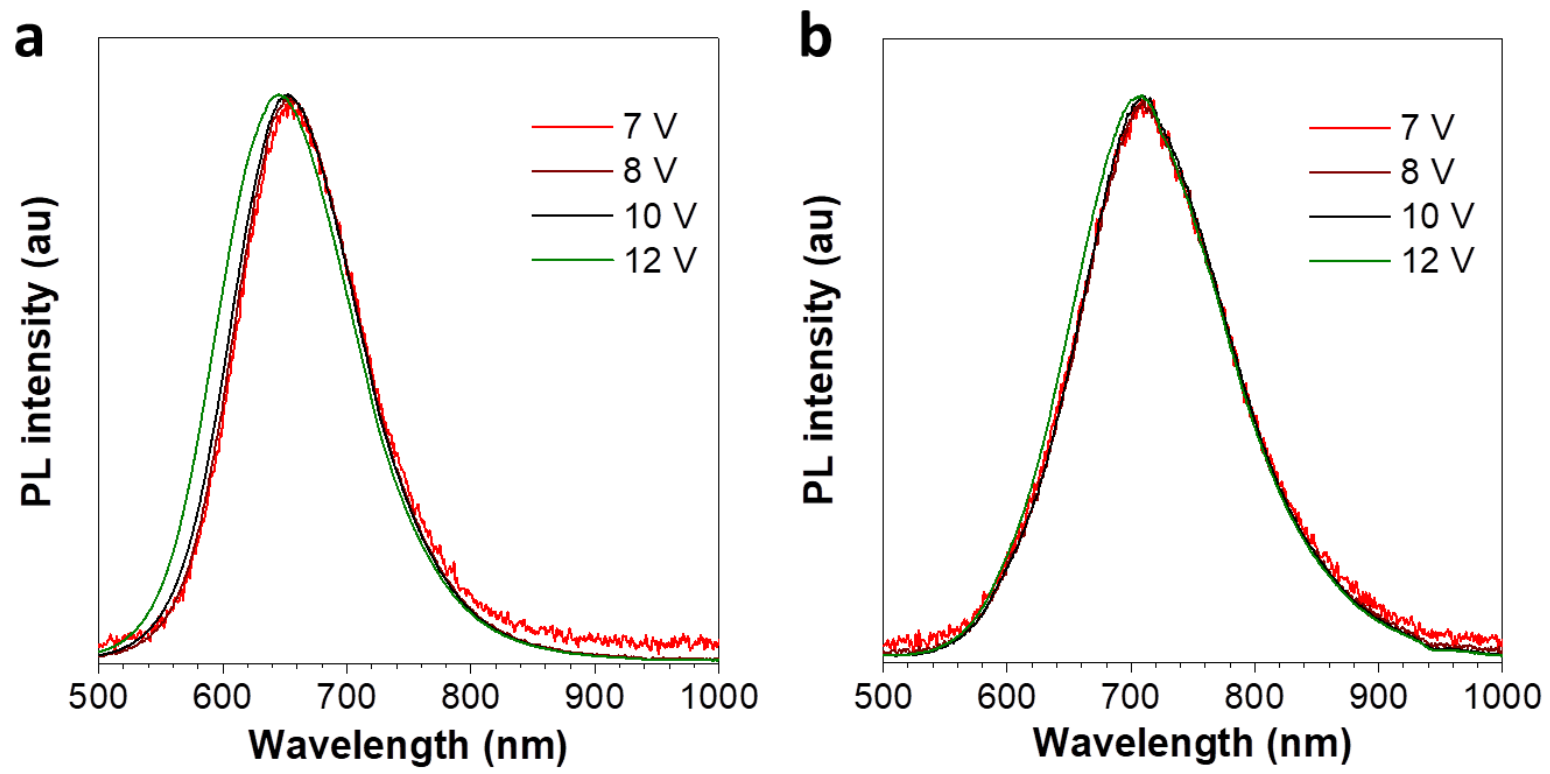

Figure S5. Electroluminescence (EL) curves at different voltages of devices using (a) DCMa and (b) DCIs as emitter, respectively.
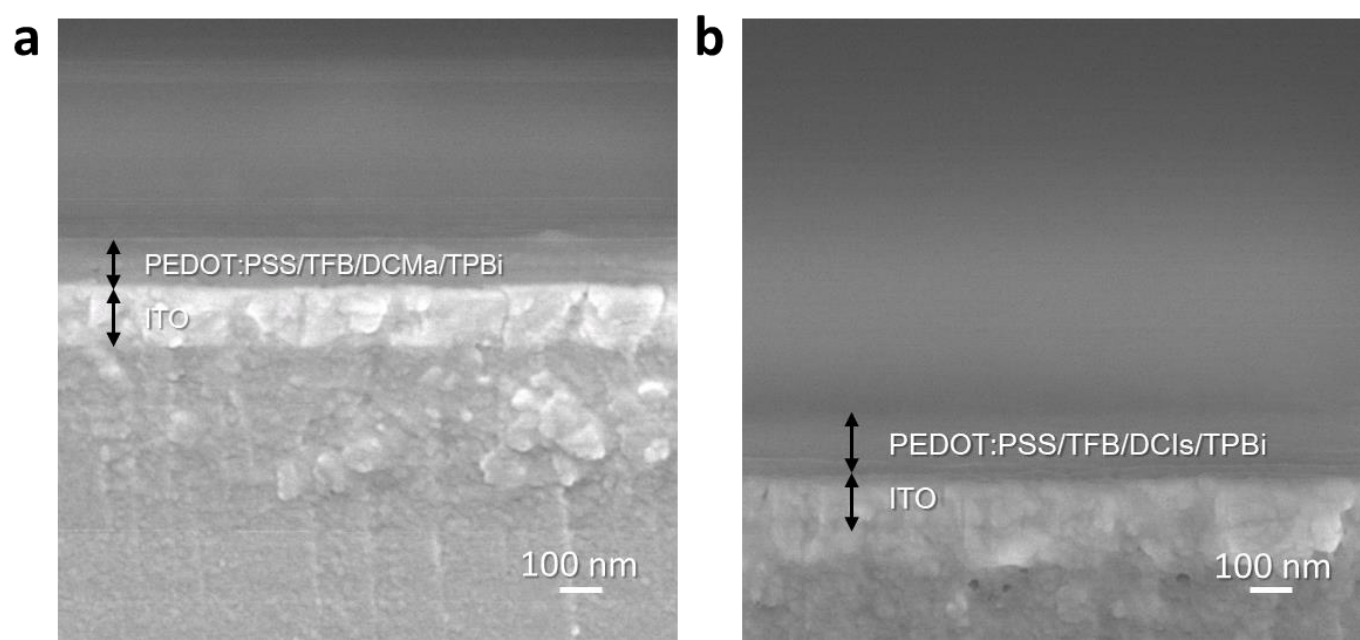

Figure S6. SEM image of cross section of OLED devices using (a) DCMa and (b) DCIs as the emissive layer without $\mathrm{LiF} / \mathrm{Al}$ layer.
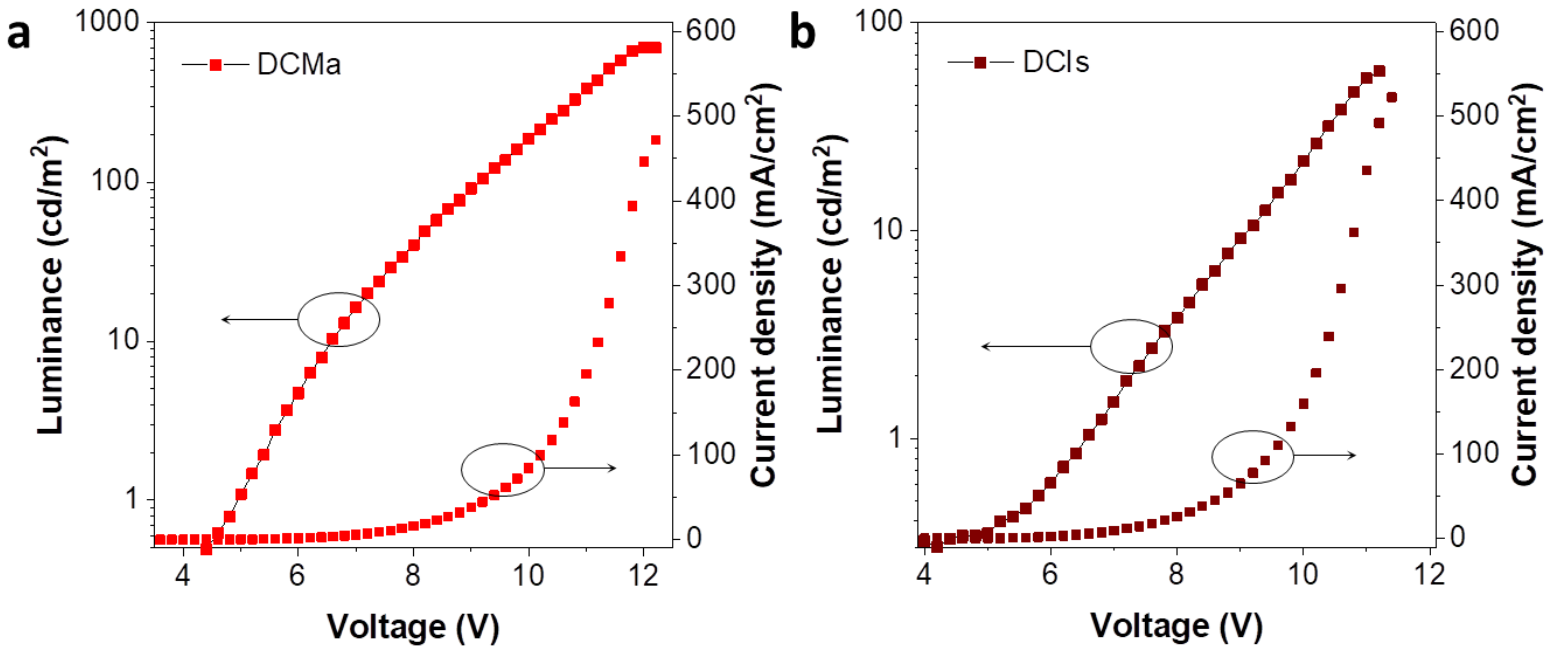
Figure S7. Current density-voltage-luminance (J-V-L) characteristics of (a) DCMa and (b) DCIs.
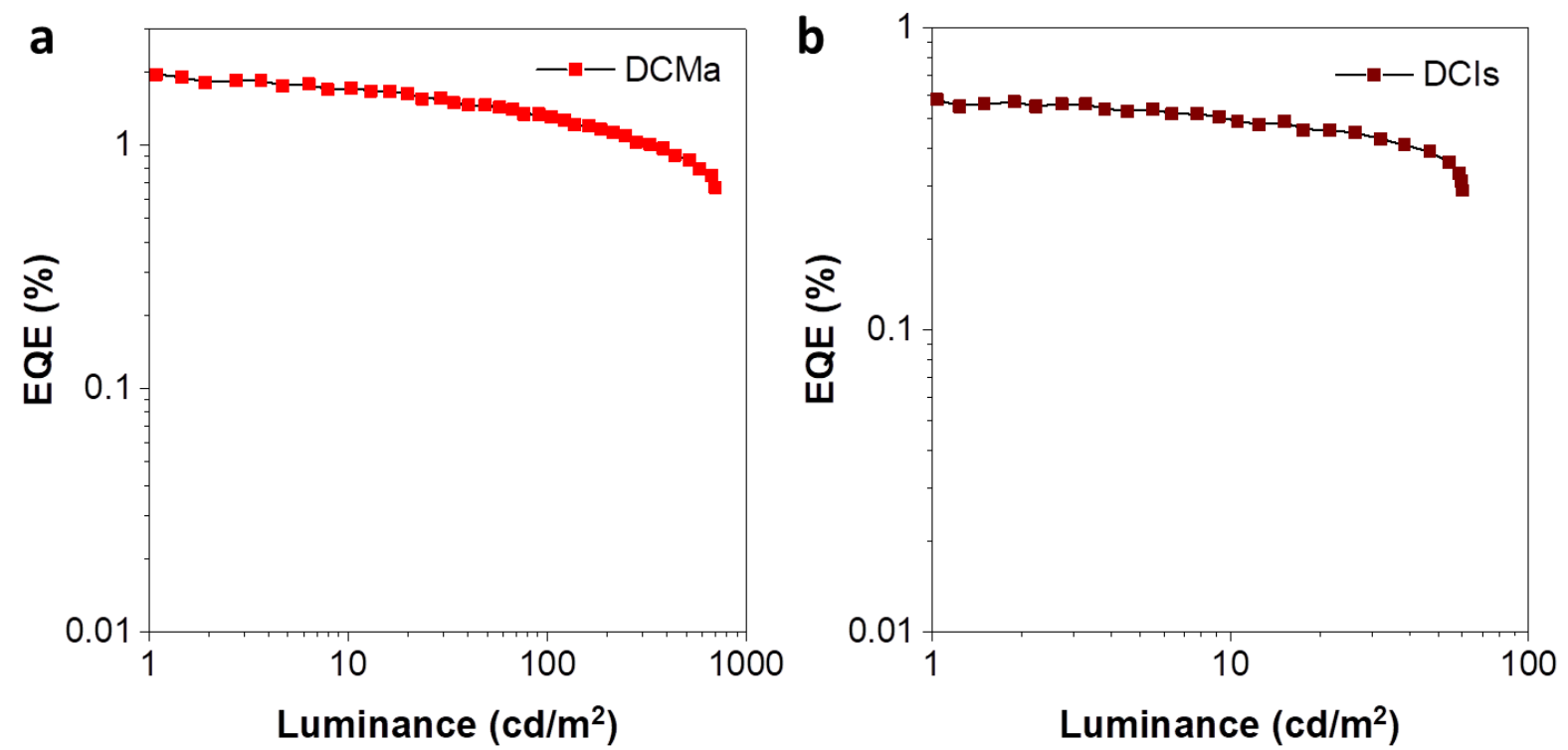

Figure S8. Curve of EQE versus luminance of devices using (a) DCMa and (b) DCIs as emitter, respectively.

Table S2. EL performance of solution-processed non-doped OLED devices.

\begin{tabular}{ccccc}
\hline Emitter & $\lambda_{\text {EL }}[\mathrm{nm}]$ & $V_{\text {on }}[\mathrm{V}]^{\mathrm{a})}$ & $\mathrm{EQE}[\%]^{\mathrm{b})}$ & $\mathrm{CIE}(\mathrm{x}, \mathrm{y})^{\mathrm{c})}$ \\
\hline $\mathrm{DCMa}$ & 652 & 5.0 & 1.95 & $0.63,0.36$ \\
DCls & 711 & 6.6 & 0.58 & $0.64,0.35$ \\
\hline
\end{tabular}

a) $V_{\text {on }}$ is the turn-on voltage at $1 \mathrm{~cd} \cdot \mathrm{m}^{-2}$;

b) Maximal external quantum efficiency;

c) $\mathrm{CIE}$ coordinates at $10 \mathrm{~mA} \cdot \mathrm{cm}^{-2}$. 